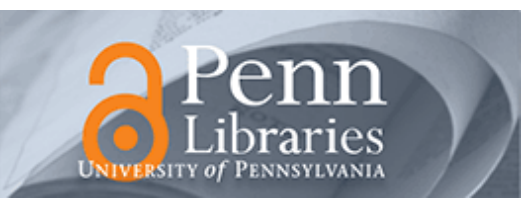

University of Pennsylvania

ScholarlyCommons

October 2001

\title{
Afferent Stream Integration in a Model of the Nucleus Accumbens
}

\author{
John A. Wolf \\ University of Pennsylvania,wolfjo@mail.med.upenn.edu \\ Lee F. Schroeder \\ University of Pennsylvania \\ Diego Contreras \\ University of Pennsylvania, diegoc@mail.med.upenn.edu \\ Leif $\mathrm{H}$. Finkel \\ University of Pennsylvania, leif@neuroengineering.upenn.edu
}

Follow this and additional works at: https://repository.upenn.edu/be_papers

\section{Recommended Citation}

Wolf, J. A., Schroeder, L. F., Contreras, D., \& Finkel, L. H. (2001). Afferent Stream Integration in a Model of the Nucleus Accumbens. Retrieved from https://repository.upenn.edu/be_papers/35

Copyright 2001 IEEE. Reprinted from Proceedings of the 23rd Annual International Conference of the IEEE Engineering in Medicine and Biology Society 2001 Volume 1, pages 796-801.

Publisher URL: http://ieeexplore.ieee.org/xpl/tocresult.jsp?isNumber=21918\&page=15

This material is posted here with permission of the IEEE. Such permission of the IEEE does not in any way imply IEEE endorsement of any of the University of Pennsylvania's products or services. Internal or personal use of this material is permitted. However, permission to reprint/republish this material for advertising or promotional purposes or for creating new collective works for resale or redistribution must be obtained from the IEEE by writing to pubs-permissions@ieee.org. By choosing to view this document, you agree to all provisions of the copyright laws protecting it.

This paper is posted at ScholarlyCommons. https://repository.upenn.edu/be_papers/35

For more information, please contact repository@pobox.upenn.edu. 


\title{
Afferent Stream Integration in a Model of the Nucleus Accumbens
}

\author{
Abstract \\ The Nucleus Accumbens (Nacc) receives convergent input from a number of structures including \\ prefrontal cortex, hippocampus, and amygdala, as well as substantial dopaminergic modulation. The \\ principle cell in the Nacc, the medium spiny projection neuron (MSP), has bistable properties that have \\ been proposed as a primary mediator of the integrative properties in the Nacc. In order to determine the \\ minimum biophysical properties required to generate a nonlinear "bistable" membrane potential, we \\ constructed a 29 compartment MSP cell in GENESIS. This included an inward rectifying $\mathrm{K}^{+}\left(\mathrm{K}_{\mathrm{IR}}\right)$, an $\mathrm{A}^{-}$ \\ current $\mathrm{K}^{+}, \mathrm{HVA} \mathrm{Ca}^{2+}$ and suitable fast $\mathrm{Na}^{+}$and delayed rectifier $\mathrm{K}^{+}$channels. Various amounts and \\ distributions of afferent input were examined to determine the amount of coincident input required to \\ move the membrane from the "down" state $(-85 \mathrm{mV})$ to the "up" state $(-60 \mathrm{mV})$ and to fire the cell. The \\ number of synchronous afferents required was substantially higher than previously estimated. We \\ modeled the effect of dopaminergic modulation by increasing the conductance of the $\mathrm{KIR}_{\mathrm{R}}$ and $\mathrm{Ca}^{2+}$ \\ channels, demonstrating that the response to input is dependent on state. \\ In parallel studies, in vivo extracellular recordings were obtained from Nacc and neocortex in anesthetized \\ mice. Simultaneous recordings revealed that cortex and Nacc oscillate in synchrony. Lesions that disrupt \\ slow oscillations in the cortex alter rhythmicity in the Nacc, suggesting interdependency of the \\ mechanisms generating bistability.
}

\section{Keywords}

Nucleus Accumbens, modeling, medium spiny neurons, in vivo, electrophysiology

\section{Comments}

Copyright 2001 IEEE. Reprinted from Proceedings of the 23rd Annual International Conference of the IEEE Engineering in Medicine and Biology Society 2001 Volume 1, pages 796-801.

Publisher URL: http://ieeexplore.iee.org/xpl/tocresult.jsp?isNumber=21918\&page=15

This material is posted here with permission of the IEEE. Such permission of the IEEE does not in any way imply IEEE endorsement of any of the University of Pennsylvania's products or services. Internal or personal use of this material is permitted. However, permission to reprint/republish this material for advertising or promotional purposes or for creating new collective works for resale or redistribution must be obtained from the IEEE by writing to pubs-permissions@ieee.org. By choosing to view this document, you agree to all provisions of the copyright laws protecting it. 


\title{
AFFERENT STREAM INTEGRATION IN A MODEL OF THE NUCLEUS ACCUMBENS
}

\author{
J.A.Wolf ${ }^{1}$, L.F. Schroeder ${ }^{2}$, D.Contreras ${ }^{1}$, L.H.Finkel ${ }^{2}$ \\ ${ }^{3}$ Department of Neuroscience and ${ }^{2}$ Bioengineering, University of Pennsylvania, Philadelphia, PA, USA
}

\begin{abstract}
The Nucleus Accumbens (Nacc) receives convergent input from a number of structures including prefrontal cortex, hippocampus, and amygdala, as well as substantial dopaminergic modulation. The principle cell in the Nacc, the medium spiny projection neuron (MSP), has bistable properties that have been proposed as a primary mediator of the integrative properties in the Nacc. In order to determine the minimum biophysical properties required to generate a nonlinear "bistable" membrane potential, we constructed a 29 compartment MSP cell in GENESIS. This included an inward rectifying $\mathrm{K}^{+}\left(\mathrm{K}_{1 \mathrm{R}}\right)$, an A-current $\mathrm{K}^{+}$, $\mathrm{HVA} \mathrm{Ca}^{2+}$ and suitable fast $\mathrm{Na}^{+}$and delayed rectifier $\mathrm{K}^{+}$channels. Various amounts and distributions of afferent input were examined to determine the amount of coincident input required to move the membrane from the "down" state $(-85 \mathrm{mV})$ to the "up" state $(-60 \mathrm{mV})$ and to fire the cell. The number of synchronous afferents required was substantially higher than previously estimated. We modeled the effect of dopaminergic modulation by increasing the conductance of the $\mathrm{K}_{\mathrm{IR}}$ and $\mathrm{Ca}^{2+}$ channels, demonstrating that the response to input is dependent on state.

In parallel studies, in vivo extracellular recordings were obtained from Nacc and neocortex in anesthetized mice. Simultaneous recordings revealed that cortex and Nacc oscillate in synchrony. Lesions that disrupt slow oscillations in the cortex alter rhythmicity in the Nacc, suggesting interdependency of the mechanisms generating bistability.
\end{abstract}

Keywords - Nucleus Accumbens, modeling, medium spiny neurons, in vivo, electrophysiology

\section{INTRODUCTION}

The nucleus accumbens ( $\mathrm{Nacc}$ ) is an integrative center located in the subcortical basal forebrain, where it receives major afferent projections from prefrontal cortex, hippocampus, and amygdala. The Nacc has been implicated in a number of disorders including addiction and schizophrenia. It has been proposed that information from the prefrontal cortex (PFC) is modulated by the afferent inputs from the hippocampus and the amygdala, and that a disturbance in this information processing underlies the symptomatology of schizophrenia.[1]

The nucleus is composed almost entirely of inhibitory cells, medium spiny projection (MSP) neurons, which comprise $\sim 95 \%$ of the cell population. MSP neurons are interconnected both by gap junctions and $\mathrm{GABA}_{\mathrm{A}}$ synapses. The MSP cells display an unusual "bistable" 2-state membrane potential. In the "down" state (resting potential $-85 \mathrm{mV}$ ) cells are restricted from firing; in the "up" state (resting potential $\sim-60 \mathrm{mV}$ ) cells can be depolarized into firing [2-4]. In the dorsal striatum, state transitions occur in a coordinated manner among coupled MSP neurons in local anatomical microzones [5].

Dopamine modulates the physiological properties of MSP cells and their ability to switch between states. The accumbens receives a massive dopaminergic input from the ventral tegmentum, and together with prefrontal cortex, Nacc controls the release of dopamine by ventral tegmentum. At the cellular level, dopamine modulates several intrinsic currents, including a persistent $\mathrm{Na}^{+}$channel, L-type $\mathrm{Ca}^{2+}$ channels and an anomalous inward rectifying potassium current, $\mathrm{K}_{\mathrm{IR}}$. Malenka and colleagues [6] have postulated that dopamine acts to stabilize MSP neurons in either the up or down states by effectively increasing the energy barrier to state transitions. Grace and colleagues [1] have proposed that these transitions between the "up" and "down" states allow MSP neurons to act as a gate controlling information flow along the reentrant loop linking prefrontal cortex, ventral striatum and thalamus. They propose that input from the hippocampus opens the gate by depolarizing MSP neurons into the up state, at which point information from the prefrontal cortex is allowed through.

It is attractive to consider that the two-state membrane potential of the MSP neuron serves as a gating mechanism, but other interpretations are possible. It is conceivable, for example, that the hippocampal and amygdala inputs serve to "route" information through the Nacc, with the pattern of hippocampal input determining how the prefrontal cortical input to Nacc is coupled back to PFC. Recent experimental data suggests that transitions between MSP bistable states can be driven by the PFC input itself [7]. Thus, the accumbal networks might be envisioned as carrying out some form of afferent stream integration on the combined inputs from PFC, hippocampus, amygdala and elsewhere, with the result of this computation determining the information content of the accumbal output as well as its neuronal targets. In such a mechanism, the accumbens would fuse multiple information streams: the PFC providing goal-directed information, the hippocampus providing current environmental context, and the amygdala the affective valence. A less sanguine possibility is that the bistable state is a result of the anesthetic state, and does not contribute to functional behavior in the awake animal. Simultaneous recordings from the PFC and Nacc in the awake animal are required to test this last hypothesis.

As a first step towards understanding these issues, we wish to examine the cellular mechanisms operating in MSP neurons. In particular, how do the specific afferent spike trains impingent upon the network determine transitions between states and the firing of MSP neurons. We are motivated by several mechanistic questions: 1) What is the biophysical mechanism underlying the bistable membrane potential of MSP neurons? 2) What determines transitions between membrane states - i.e., are there spatial and/or temporal requirements on the afferent spike trains? 3) Can a model determine the role of PFC versus hippocampal inputs 
it:

regarding state transitions and MSP firing? 4) What types of computations might be carried out by such a network? We seek to develop a biophysical-level model of a single MSP neuron with which one could investigate the number of inputs required to switch cell state, and the effect of dopaminergic modulation on this process.

The state of the cells in the accumbens depends on the temporal properties of the input stream. If the inputs are strongly synchronized, this may drive the oscillations of the MSP cells. A potential confound of the in vivo study of these issues is the synchronizing effect of some anesthesias on the cells that have efferents to the Nacc, such as the cortex. To begin the process of understanding how cortical inputs effect the Nacc and possibly the state transitions, we simultaneously recorded multiple-site extracellular field potentials in the cortex and the Nacc of the mouse under anesthesia.

\section{METHODOLOGY}

We constructed a reduced model of an MSP neuron using GENESIS [8]. In vivo, MSP neurons have small to medium somata $(15.8 \pm 2.8 \mu \mathrm{m})$ and a multipolar or fusiform shape from which 3-6 primary dendrites arise (diameter, $1-2 \mu \mathrm{m}$ ). The first dendritic branch point arises $20-30 \mu \mathrm{m}$ out from the soma, and the secondary and higher-order dendrites are densely covered with spines (the primary dendrites are smooth) [7]. The cells are electrotonically compact, with an input resistance measured at $217 \pm 33 \mathrm{M} \Omega$ in whole cell patch clamp [7]. These properties are captured in a 29compartment model, with 4 primary dendrites.

MSP cells contain a number of active currents, including voltage-dependent $\mathrm{Na}^{+}$and delayed rectifier channels, a persistent $\mathrm{Na}^{+}$current, a variety of $\mathrm{Ca}^{2+}$ channels $(\mathrm{L}, \mathrm{N}, \mathrm{P} / \mathrm{Q}$, $R$ and $T$ ), and $a K_{A}$ current. Most interestingly, cells contain an unusual, inward-rectifying $\mathrm{K}^{+}$channel $\left(\mathrm{K}_{\mathrm{IR}}\right)$ that is active at hyperpolarized potentials [4]. The $K_{I R}$ channel underlies the unusually hyperpolarized "down" state $(\sim-85 \mathrm{mV})$. This channel has extremely fast kinetics, and does not inactivate-rather, it closes with minimal depolarization, effectively shutting off by $-65 \mathrm{mV}$. The $\mathrm{K}_{\mathrm{IR}}$ current flows outward (hyperpolarizing) for all voltages above the reversal potential $(-90 \mathrm{mV})$; the channel is called an "inward rectifier" because it preferentially allows inward current at membrane potentials more negative than $-90 \mathrm{mV}$.

$K_{I R}$ kinetics were adapted from Hodgkin-Huxley $\alpha$ and $\beta$ parameters from Hayashi and Fishman's studies of $K_{\mathrm{IR}}$ in Aplysia [9], and modified until they generated an I-V curve that matched that published by Wilson [3]. Wilson generated his I-V curve from Hagiwara and Takahasi's data on $\mathrm{K}_{\mathrm{IR}}$ in starfish eggs [10], together with membrane resistances as determined in spiny neostriatal cells. Clearly, extrapolating from invertebrates, compensating for temperature differences, and other factors make our $\mathrm{K}_{\mathrm{IR}}$ current model an approximation to this current in accumbal cells.

The Hodgkin-Huxley parameters for modeling the $\mathrm{K}_{\mathrm{A}}$ channel were based on those used by Traub [11] for hippocampal pyramidal cells, and modified to fit the data reported by Gabel and Nisenbaum [12] in rat neostriatum. As with the $\mathrm{K}_{\mathrm{IR}}$ current, we explored the effects of small shifts in the $K_{A} \mathrm{I}-\mathrm{V}$ curve on the bistability of the membrane potential. These potassium currents serve as the anchors for the two membrane states. Transition between states depends upon driving the potential past the crossing-point of the curves. In our studies, we found that one of the critical elements for attaining bistability is that the $\mathrm{K}_{\mathrm{IR}}$ channel close at a slightly more hyperpolarized voltage than that at which the $K_{A}$ channel begins to substantially open.

We modeled the effects of dopamine on our MSP neuron by doubling $K_{I R}$ from its baseline value of $50 \mathrm{~S} / \mathrm{m}^{2}$ and quadrupling the $\mathrm{L}$-type $\mathrm{Ca}^{2+}$ conductance from its baseline value of $5 \mathrm{~S} / \mathrm{m}^{2}$ [13]. These baseline conductances correspond to the low-end values reported in the literature, and the increase due to dopaminergic stimulation corresponds to the high-end values observed experimentally $[13,14]$. The L-type $\mathrm{Ca}^{2+}$ channel was constructed based on data reported for the high-voltage activated channel [13]. Dopaminergic effects on AMPA conductances were not included. To investigate the up state, the cell was injected with $0.6 \mathrm{nA}$ current for $50 \mathrm{~ms}$. Separate simulations show that this current' corresponds to approximately 6000 synaptic inputs per second. To investigate the down state, the cell was stimulated with a barrage of 500 synaptic inputs per second, and an additional barrage of 100 inputs delivered over a 10 ms interval.

In vivo electrophysiology was performed on C57/BL6 mice anesthetized with a $1: 1$ mixture of ketamine $(200 \mathrm{mg} / \mathrm{kg})$ xylazine $(40 \mathrm{mg} / \mathrm{kg})$ and placed in a stereotaxic device. Bipolar tungsten-recording electrodes were placed in the cortex and Nacc ( $\sim 1$ Mohm impedance). A cortical lesion was made with $\mathrm{AgNO}_{3}$ crystals in a large area of the frontoparietal cortex in order to disrupt cortical synchronization. . Data was stored digitally using a CEDI 401 system and analyzed offline with IGOR and Matlab packages.

\section{RESULTS}

\section{Intrinsic Properties Underlying the 2-State Potential}

Incorporating the $\mathrm{K}_{\mathrm{IR}}$ and $\mathrm{K}_{\mathrm{A}}$ currents into our 29compartment MSP neuron model shows that these two currents (along with the standard $\mathrm{Na}^{+}$and $\mathrm{K}_{\mathrm{DR}}$ currents), are sufficient to generate the 2-state, bistable membrane potential. Figure 1 shows the results of a series of ten current injections applied to the soma of the modeled MSP neuron. The current injections were graduated from 0.2 to $0.5 \mathrm{nA}$ in equal increments, each lasting $40 \mathrm{~ms}$. Increasing currents lead to greater depolarization, but there is a clear non-linearity in the steady-state voltage level reached, with a bifurcation into an "up" and "down" state. The highest level of current injection leads to the generation of action potentials from the "up" state. These results closely correspond to intracellular recordings from accumbal cells.

In the down state, small current injections are countered by the $\mathrm{K}_{\mathrm{IR}}$ current, which acts to keep the potential near the reversal potential of $-90 \mathrm{mV}$. Larger injections are able to depolarize the membrane, and begin to close $\mathrm{K}_{\mathrm{IR}}$. Closing the $\mathrm{K}_{\mathrm{IR}}$ channels increases membrane resistance, thus increasing current generates a nonlinear increase in depolarization. This nonlinearity helps propel the potential from the "down" state 
to the "up" state. However, sufficient depolarization begins to open $\mathrm{K}_{\mathrm{A}}$ channels, which counter the injected currents and tend to stabilize the potential near $-60 \mathrm{mV}$. Increasing depolarization opens more $\mathrm{K}_{\mathrm{A}}$ channels, decreasing membrane resistance, and thus reducing the induced potential change. A disproportionate increase in the injected current is needed to depolarize the cells the last few millivolts above $60 \mathrm{mV}$ required to generate action potentials. The two potassium channels, with their offset and opposing voltage dependencies, thus create "energy barriers" to depolarizing the membrane potential, which defines the two membrane potential states. Continued current injection is required to keep the membrane in the "up" state; when the current is terminated, the potential quickly falls back to the "down" state.

Wilson [2] has stressed that the bistable membrane potential is not an intrinsic membrane property, but that ongoing synaptic input is required to maintain the cell in the up state. In the model used here, $\mathrm{K}_{\mathrm{IR}}$ and $\mathrm{K}_{\mathrm{A}}$ act to maintain the potentials that define the down and up states, respectively. However, the membrane potential also reflects the input synaptic current and thus assumes a continuum of values. It is important to note that MSP neurons contain a number of other intrinsic conductances, many or all of which may contribute to the stability of the membrane states. We do not claim that $\mathrm{K}_{\mathrm{IR}}$ and $\mathrm{K}_{\mathrm{A}}$ solely determine the bistability, rather, just that alone they are sufficient to provide a major component of bistability.

In vivo, the striking nature of the bistable states is their persistence over $500 \mathrm{~ms}$ or longer, and we therefore wondered whether longer lasting currents, such as L-type $\mathrm{Ca}^{2+}$ channels and/or NMDA receptor currents might allow the cell to persist in the up state in the absence of synaptic input. We stimulated the cell with a synchronized barrage of synaptic inputs arriving within a $5 \mathrm{~ms}$ time window delivered at 30 $\mathrm{Hz}$. This stimulation frequency was chosen to correspond to a typical firing rate of delay-period neurons in prefrontal cortex (see discussion). However, our studies revealed that even in the presence of these additional currents, the cell was not able to remain in the up state. Activation of NMDA currents should in principle be able to maintain the up state, however the membrane in the up state is not sufficiently depolarized to release NMDA receptors from the $\mathrm{Mg}^{2+}$ block, and simulation of NMDA activation confirmed this.

We also studied the effect of applying a synchronized barrage of AMPA-mediated synaptic inputs to the cell. Inputs were delivered in a barrage to the most proximal compartment on a single dendrite, with spike times distributed in a Gaussian distribution. Increasing in the $K_{I R}$ current, such as would occur in the presence of dopamine (see below), increases the number or temporal synchrony of inputs required to switch to the up state. The data shows that on the order of 100-450 roughly synchronous inputs are required for the transition. This number can be compared to the estimate speculated by Lopes da Silva and colleagues [7] who suggested an order of magnitude less inputs would be sufficient. Our findings reflect the effects of the $K_{\mathrm{IR}}$ channel as well as some aspects of dendritic geometry. The required

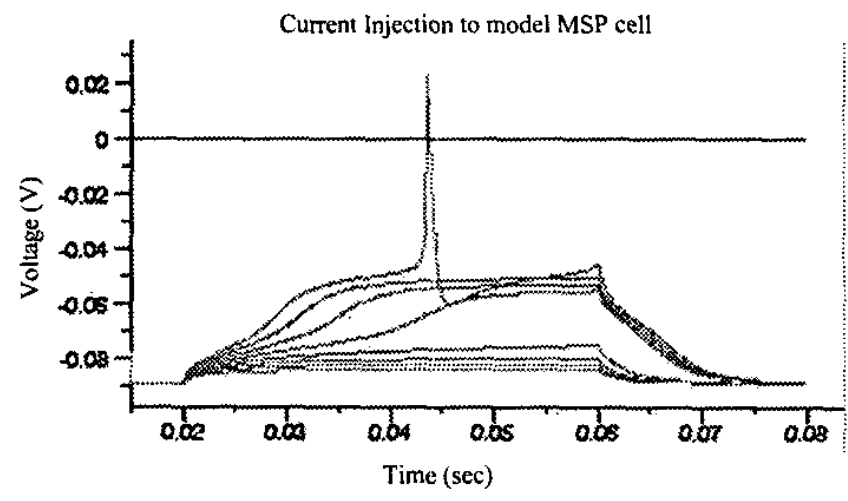

Fig.1 Membrane potential response of the cell to linear increases in current injection. The non-linearity of the response is demonstrated by the appearance of two distinct states.

number of inputs increases roughly linearly with the temporal dispersion, but with a small slope, so that the density of $\mathrm{K}_{\mathrm{IR}}$ current is the major factor in determining how many inputs are required.

\section{Dopaminergic Modulation}

The modulatory effects of dopamine arise from effects on a range of membrane channels together with effects on both glutamatergic and GABAergic synaptic transmisssion [6]. A variety of subtypes of dopamine receptors (D1-D5) are expressed on different cell types. However, all dopamine receptors act via $G$ protein signaling to modulate adenylyl cyclase: D1 receptors activate adenylyl cyclase, whereas D2 and D3 receptors inhibit adenylyl cyclase [6]. By increasing adenylyl cyclase, D1 agonists decrease the peak $\mathrm{Na}^{+}$current and both $\mathrm{N}$ and $\mathrm{P} / \mathrm{Q} \mathrm{Ca}^{2+}$-currents, and increase both $\mathrm{K}_{\mathrm{IR}}$ and L-type $\mathrm{Ca}^{2+}$ currents.

In cellular recordings, dopamine has been reported to be capable of either exciting or inhibiting MSP activity. These differences may arise, in part, from the activation of different receptor subtypes. However, conflicting results have been obtained in studies from the same lab in which both DI and D2 receptors are activated (some carried out in D3 knock-out mice) [16]. Nicola, Surmeier and Malenka [6] suggest that the reason for these conflicting findings is that the effect of dopamine depends on the state (up vs. down) of the MSP neuron. They propose that in the down state, D1 activation suppresses excitation by depressing the $\mathrm{Na}^{+}$current and enhancing the $K_{\mathrm{IR}}$ current. For cells in the up state, where $\mathrm{K}_{\mathrm{IR}}$ is deactivated, $\mathrm{D} 1$ input increases the L-type $\mathrm{Ca}^{2+}$ current and NMDA currents (but suppresses AMPA currents). D1 activation is thought to stabilize neurons in their current state and to increase the barrier to switching states [6]. This is supported by in vivo experiments showing that dopamine suppresses background activity while enhancing goal-directed activity [17].

As shown in figure 2, the effect of dopamine on the MSP cell depends on cell state. In the down state, the response to the input is suppressed by dopamine. However, in the up state, dopamine causes a marked increase in firing frequency in response to this same current injection. These results 

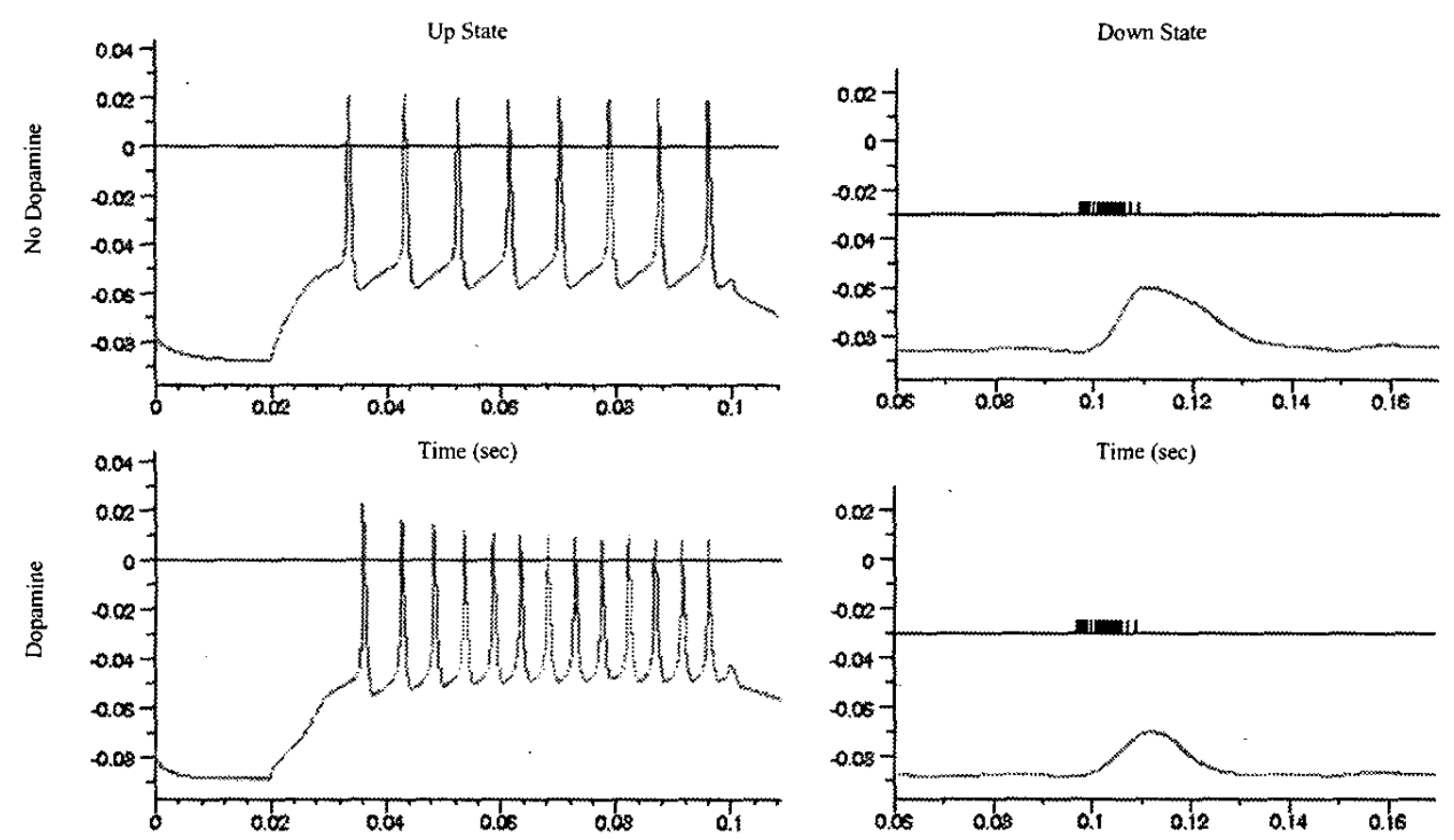

Fig.2 Response of the cell to current injection (left) and synaptic input (right) in with and without dopamine. A differential effect is observed depending on the state of the cell. While in the up state, dopamine increases firing rate (lower left), whereas in the down state it decreases the effect of the input on the membrane potential.

correspond to those obtained experimentally by HernandezLopez and colleagues [18] who showed that dopamine applied to the up state decreases the interspike interval of the firing cell. Note that even though dopamine acts on both the $\mathrm{K}_{\mathrm{IR}}$ and the $\mathrm{Ca}^{2+}$ current, since each current is only active in one of the states (L-type $\mathrm{Ca}^{2+}$ in the up state, $\mathrm{K}_{\mathrm{IR}}$ in the down state), the net effect of dopamine is state-dependent. Increased $K_{I R}$ is responsible for suppressing the response to synaptic input in the down state, and increased $\mathrm{Ca}^{2+}$ current leads to greater excitability in the up state. We have not incorporated dopaminergic effects on synaptic conductances, which might further increase the observed effect, and may compensate for the accommodation in spike amplitudes seen in figure 2.

\section{In Vivo Elecrophysiology}

Recordings from the mouse cortex concurrent with the Nacc under ketamine/xylazine anesthesia show spontaneous slow oscillations. Recordings in the Nacc are correlated with a complex distribution of phase-lags across cortical sites. (Fig.3A). A small region of cortex was lesioned by applying $\mathrm{AgNO}_{3}$. Previous studies have shown that this procedure alters the pattern of cortical synchronization in areas surrounding the lesion.[19] Autocorrelogram of the Nacc reveal a shift in rhythmicity before versus after cortical lesioning. Thus, cortical synchrony modulates the rhythm present in the Nacc. (Fig.3B)

\section{DISCUSSION}

This initial model of the Nacc MSP neuron sheds some light on the questions posed above, and leads to some speculative hypotheses. The model demonstrates that the bistable membrane potential is likely mediated, in large part, by the interplay between two voltage-dependent potassium currents, $\mathrm{K}_{\mathrm{IR}}$ and $\mathrm{K}_{\mathrm{A}}$. The nonlinear transition between states requires that with increasing depolarization, $\mathrm{K}_{\mathrm{IR}}$ closes before $\mathrm{K}_{\mathrm{A}}$ opens. We used identical maximal conductance values for these currents $\left(100 \mathrm{~S} / \mathrm{m}^{2}\right)$, so that membrane resistance is roughly equivalent in the up and down state. We examined the amount of simultaneous input that would be required to move the celis from the "down" state to the "up" state. Lopes da Silva and colleagues provide a motivation for this analysis in their comprehensive review [7]. Making best-guess approximations for membrane resistances and single-afferent postsynaptic currents, they calculate that $\sim 15-50$ concurrent input spikes would be required to switch cells from the down to the up state. They rued however, that this back-of-theenvelope calculation could not take account of effects due to the $\mathrm{K}_{\mathrm{IR}}$ current, dendritic geometry, and other complicating factors. All of the received inputs might have to occur within a narrow time window $(\sim 2-5 \mathrm{msec})$ in order to kick the cell into the up state. Our results indicate that depending on the level of $\mathrm{K}_{\mathrm{IR}}$ present in the dendrites, 100-450 simultaneous inputs would be necessary to move a cell into the up state.

We were unable to maintain the cell in the up state in the absence of continued stimulation. Thus, in agreement with Wilson [2], we find that the up state is not an intrinsically stable membrane state, but requires ongoing stimulation. Of particular interest is whether PFC inputs on their own are capable of maintaining the MSP in the up state. We found this not to be the case: stimulation with a sufficient number of synchronized inputs delivered at $30 \mathrm{~Hz}$ switches the cell to 

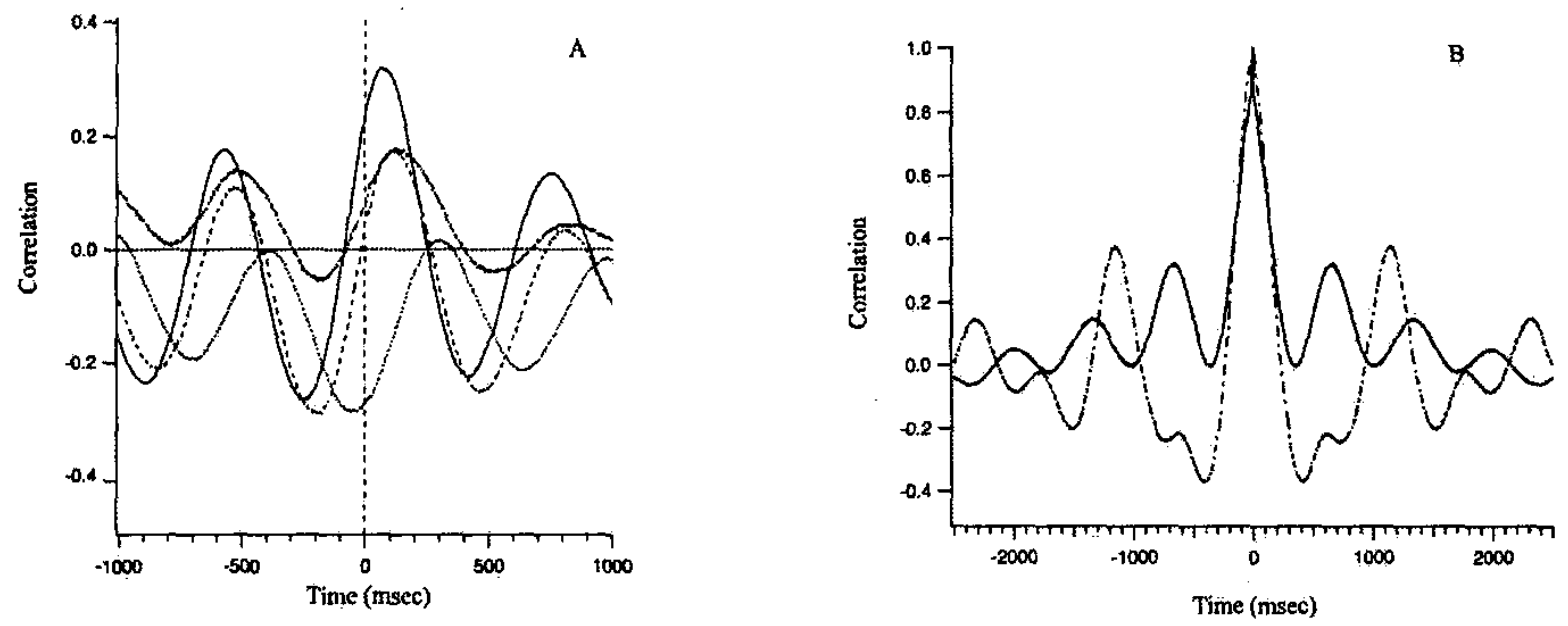

Fig. 3 Correlation of activity in the Nacc and cortex. 3A shows the cross-correlation between four different cortical electrodes and the Nacc - along with the correlation there is a phase lag between different areas of the cortex in relation to the accumbens. 3B shows the results of an auto-correlogram of the Nacc before (solid line) and after (dashed line) cortical lesion. After cortical lesion there is a shift in the timing of the oscillations in the Nacc.

the up state, but the potential falls back to the down state before the next input barrage $(33 \mathrm{~ms})$. In our simulations, the up state voltage $(\sim-60 \mathrm{mV})$ was insufficient to maintain NMDA activation, and the L-type $\mathrm{Ca}^{2+}$ current implemented here was similarly unable to generate sufficient current to maintain the up state. Maintenance of the up state therefore appears to require ongoing input to the cell. However, it remains to be determined whether a detailed model of the effects of dendritic spines, or incorporation of network properties among coupled MSP cells might contribute to the persistence of the up state.

Our investigation of the effects of dopamine on cell response are in agreement with the evidence put forward by Malenka and colleagues [6] and with recent modeling studies in prefrontal cortex [20]. We find that dopamine acts to stabilize cells in their present state, or to increase the barrier to state transitions. In the down state, dopamine accentuates the $\mathrm{K}_{\mathrm{TR}}$ current, damping out the effects of afferent stimulation; in the up state, dopamine accentuates the L-type $\mathrm{Ca}^{2+}$ current, which amplifies cell responses to input. We have only incorporated D1 receptor effects, and thus the effect could be further dissociated according to synaptic location or cell type. The ability of dopamine to change cell gain in a state-dependent manner may allow the cell to respond in a context-dependent manner.

We have begun to investigate the correlation of activity between areas that have afferent input to the Nacc and the Nacc itself. It has previously been demonstrated that there is a large projection from the pre-limbic cortex to the Nacc. To examine the relationship between the oscillations in the cortex and those in the Nacc under anesthesia, we performed simultaneous extracellular field potential recordings and then lesioned the cortex with $\mathrm{AgNO}_{3}$. There was a large correlation between the multiple sites recorded in the cortex and the field potentials in the Nacc. The rhythmicity in the Nacc was altered significantly by the lesion of the cortex, presumably due to the alteration in synchronization of the of the cells projecting to the Nacc. These results suggest that the mechanisms generating the slow oscillations in the cortex and the Nacc are intimately related. Given the importance of the oscillatory states in the Nacc for information processing, it is critical that we understand the generation of these oscillations.

\section{Afferent Stream Integration in the Accumbens:}

The biophysical properties of MSP cells provide the underpinning for how the Nacc responds to converging afferent inputs. MSP cells are presumed to mutually inhibit each other via GABAergic synapses, however, they also are coupled via gap junctions. Connors and colleagues have shown that a similar network architecture is capable of rapid synchronization [21]. They investigated low-threshold spiking interneurons in neocortex, which are interconnected by both GABAergic synapses and gap junctions, and showed that such networks could be synchronized by the application of neuromodulators, such as acetylcholine. Hopfield [22] has simulated this type of network dynamics, and has shown that the transient synchrony emerging and re-emerging over time among different subpopulations of neurons can carry out interesting computations, including recognition and generalization.

One possible conception of the computation performed by Nacc is a prediction of whether an animals current trajectory (motor or cognitive) is directed towards the appropriate goal. This prediction might be expressed through the pattern of synchronously firing MSP cells. The process of pattern integration might be carried out by associative memory mechanisms that learn to associate patterns across the input streams. A second class of possible mechanisms would allow the accumbens to compute a prediction of whether the recent inputs received from the hippocampus are progressing towards a goal defined by the PFC. Such a network would more closely resemble a Kalman filter, and the implementation might resemble that suggested for predictive coding in the cortico-thalamic loop [23].

It is interesting to speculate that dopamine might serve to transiently synchronize accumbal networks. Grace and colleagues [1] have evidence that gap junctional coupling between MSP cells is controlled by NO (nitric oxide) released in response to dopamine. Selective modulation by dopamine 
might lead to the emergence of transiently synchronized MSP cell assemblies, which act as temporary routers on the feedback loop to prefrontal cortex. Since the release of dopamine by the VTA is controlled by the cortex, this complex reentrant circuit is capable of self-modulating the computations carried out on the basis of the combined afferent input to accumbens.

\section{CONCLUSION}

Our model of a Nacc MSP cell has allowed us to begin to examine the integration of afferent input in the Nacc. Further implementation of a network of these cells should help reveal the computations performed by the Nacc. The in vivo data presented here indicate that cortical oscillations play a significant role in determining the state of the cells under anesthesia.

\section{ACKNOWLEDGMENTS}

We would like to thank Charles Wilson for providing the code for his potassium channel models as well as a number of insightful and helpful suggestions, and Elliot Menschik for guidance on the implementation. Supported by NSF-KDI and NIH Conte Center funding.

\section{REFERENCES}

[1] Grace, A. "Gating of information flow within the limbic system and the pathophysiology of schizoprhenia." Brain Research Reviews, vol.32, pp.330-41, 2000.

[2] Wilson, C. J. and Kawaguchi, Y. "The origins of two-state spontaneous membrane potential fluctuations of neostriatal spiny neurons." J Neurosci, vol. 16, pp.2397-2410, 1996.

[3] Wilson, C. "Dendritic morphology, inward rectification, and the functional properties of neostriatal neurons." In: Single Neuron Computation. T. McKenna, J. Davis, \& S.F. Zornetzer, eds., Academic Press, Boston, pp. 141-172, 1992.

[4] Wilson, C. "The generation of natural firing patterns in neostriatal neurons." Progress in Brain Research, vol. 99, pp.277-297, 1993.

[5] Stern, E. A., Jaeger, D. \& Wilson, C. J. "Membrane potential synchrony of simultaneously recorded striatal spiny. neurons in vivo." Nature, vol.394, pp.475-8, 1998.

[6] Nicola, S. M., Surmeier, J. \& Malenka, R. C. "Dopaminergic modulation of neuronal excitability in the striatum and nucleus accumbens." Annu Rev Neurosci, vol.23, pp.185-215, 2000.

[7] Pennartz, C. M., Groenewegen, H. J. \& Lopes da Silva, F. $H$. "The nucleus accumbens as a complex of functionally distinct neuronal ensembles: an integration of behavioural, electrophysiological and anatomical data." Prog Neurobiol, vol.42, pp.719-61, 1994.

[8] Bower, J. \& Beeman, D. "The Book of GENESIS." Springer/Telos, New York, 1998.

[9] Hayashi, H. and Fishman, H.M. "Inward rectifier Kchannel kinetics from analysis of the complex conductance of Aplysia neuronal membrane." Biophysical J. vol.53, pp.747$757,1988$.
[10] Hagiwara, S. and Takahashi, K. "The anamolous rectification and cation selectivity of a starfish egg cell." $J$. Membr. Biol. vol.18, pp.61-80, 1974.

[11] Traub, R. D., Jefferys, J. G., Miles, R., Whittington, M. A. \& Toth, K. "A branching dendritic model of a rodent CA3 pyramidal neurone." $J$ Physiol (Lond), vol. 481, pp.79-95, 1994.

[12] Gabel LA, Nisenbaum ES., "Biophysical characterization and functional consequences of a slowly inactivating potassium current in neostriatal neurons. $J$ Neurophysiol. vol.79, pp.1989-2002, 1998.

[13] Avery RB, Johnston D. "Multiple channel types contribute to the low-voltage-activated calcium current in hippocampal CA3 pyramidal neurons." J Neurosci. vol.16, pp.5567-82, 1996.

[14] Bargas, J, Howe, A, Eberwine, J., Cao, Y., and Surmeier, DJ. "Cellular and molecular characterization of $\mathrm{Ca}^{2+}$ currents in acutely isolated adult rat neostriatal cells." J. Neurosci. vol.14, pp.6667-6686, 1994.

[15] Pennartz, C. M., Groenewegen, H. J. \& Lopes da Silva, F. $H$. "The nucleus accumbens as a complex of functionally distinct neuronal ensembles: an integration of behavioural, electrophysiological and anatomical data." Prog Neurobiol, vol.42, pp.719-61, 1994.

[16] Hu, X. \& White, F. "Dopamine enhances glutamateinduced excitation of rat striatal neurons by cooperative activation of D1 and D2 class receptors." Neuroscience Letters, vol.224, pp.61-65, 1997.

[17] Kiyatkin, E. A. \& Rebec, G. V. "Striatal neuronal activity and responsiveness to dopamine and glutamate after selective blockade of D1 and D2 dopamine receptors in freely moving rats." $J$ Neurosci, vol.19, pp.3594-609, 1999.

[18] Hernandez-Lopez S, Bargas J, Surmeier DJ, Reyes A, Galarraga E. "D1 receptor activation enhances evoked discharge in neostriatal medium spiny neurons by modulating an L-type $\mathrm{Ca} 2+$ conductance." J Neurosci. vol.17, pp.333442, 1997.

[19] Amzica, F., Steriade, M., "Disconnection of intracortical synaptic linkages disrupts synchronization of a slow oscillation." J Neurosci., vol.15, pp.4658-77, 1995.

[20] Durstewitz, D., Kelc, M. \& Gunturkun, O. "A neurocomputational theory of the dopaminergic modulation of working memory functions." J Neurosci, vol.19, pp.2807$22,1999$.

[21] Beierlein M, Gibson JR, Connors BW. "A network of electrically coupled interneurons drives synchronized inhibition in neocortex." Nat Neurosci., vol.3, pp.904-10, 2000.

[22] Hopfield JJ, Brody CD. "What is a moment? "Cortical" sensory integration over a brief interval." Proc Natl Acad Sci. USA vol.97: pp. 13919-24, 2000.

[23] Ahissar, E., Sosnik, R. \& Haidarliu, S. "Transformation from temporal to rate coding in a somatosensory thalamocortical pathway." Nature, vol.406, pp.302-6, 2000. 